\title{
Transcranial direct current stimulation plus concurrent activity may influence task prioritization during walking in people with Parkinson's disease - initial findings
}

This article was published in the following Dove Press journal:

Journal of Parkinsonism and Restless Legs Syndrome

\section{Christina Criminger \\ Chad Swank ${ }^{2}$ \\ Sattam Almutairi ${ }^{2}$ \\ Jyutika Mehta ${ }^{3}$}

'Department of Physical Therapy, Winston-Salem State University, Winston-Salem, NC, USA; ${ }^{2}$ School of Physical Therapy, Texas Woman's University, Dallas, TX, USA;

${ }^{3}$ Department of Communication Science and Disorders, Texas Woman's

University, Dallas, TX, USA
Correspondence: Christina Criminger Department of Physical Therapy, Winston-Salem State University, 346 FL Atkins Bldg., 60I Martin Luther King Drive, Winston-Salem, NC 27110, USA Tel + I 3367502195

Email crimingercm@wssu.edu

\begin{abstract}
Introduction: Walking for people with Parkinson's disease (PD) degrades during motor-cognitive interplay (ie, dual-task conditions). Current management of PD improves motor symptoms but inadequately addresses cognitive function, indicating a necessity for novel interventions. In this pilot study, we examined bi-hemisphere transcranial direct current stimulation (tDCS) with concurrent activity and dual-task walking in people with PD.
\end{abstract}

Methods: Participants received 3 sessions ( $\mathrm{tDCS}_{\text {sitting }}, \mathrm{tDCS}_{\text {bike, }}, \mathrm{tDCS}_{\mathrm{Wii}}$ ) of bilateral tDCS (dorsolateral prefrontal cortex; left $=$ anode, right $=$ cathode) at $2 \mathrm{~mA}$ and 1 sham session ( tDCS $_{\text {sham }}$ ). Sessions were randomized, single-blinded, and performed during medication "ON" times separated by $7 \pm 2$ days. Following each session, participants performed Timed Up and Go (TUG) single, dual-task conditions ( $\mathrm{TUG}_{\text {alone }}, \mathrm{TUG}_{\text {motor }}, \mathrm{TUG}_{\text {cognitive }}$ ).

Results: Sixteen participants with PD completed this study (mean age $=68.13 \pm 9.76$ years, Unified Parkinson's Disease Rating Scale mean=40.31 \pm 18.27 , Repeatable Battery for the Assessment of Neuropsychological Status mean=84.13 [13th percentile]). No differences were observed for TUG conditions between tDCS sessions. Dual task cost for $\mathrm{TUG}_{\text {motor }}, 14.73 \%\left(\operatorname{tDCS}_{\text {Siting }}\right)$, $17.78 \%\left(\mathrm{tDCS}_{\text {Bike }}\right), 15.97 \%\left(\mathrm{tDCS}_{\mathrm{Wii}}\right), 19.02 \%\left(\mathrm{tDCS}_{\text {Sham }}\right)$; for $\mathrm{TUG}_{\text {cognitive }}$ (walking), $20.01 \%$ $\left(\mathrm{tDCS}_{\text {Sitting }}\right), 18.7 \%\left(\mathrm{tDCS}_{\text {Bike }}\right), 31.18 \%\left(\mathrm{tDCS}_{\mathrm{wii}}\right), 20.01 \%\left(\mathrm{tDCS}_{\text {Sham }}\right)$; for $\mathrm{TUG}_{\text {cognitive }}$ (cognitive), $33.72 \%\left(\mathrm{tDCS}_{\text {Sitting }}\right), 14.99 \%\left(\mathrm{tDCS}_{\text {Bike }}\right), 4.42 \%\left(\mathrm{tDCS}_{\mathrm{wii}}\right), 19.11 \%\left(\mathrm{tDCS}_{\text {sham }}\right)$.

Conclusion: Our bi-hemisphere tDCS paired with concurrent activities did not lessen dual-task cost in participants with PD but appeared to influence task prioritization. Further investigation with a larger sample size is warranted.

Keywords: dual task interference, gait, executive function, non-invasive brain stimulation, novel task, motor-cognitive interplay

\section{Introduction}

Parkinson's disease (PD) is a neurodegenerative disease characterized by progressive motor and non-motor impairments, including cognitive deficits. Specifically, difficulty with attention and executive functions are regarded as the most common cognitive impairments in people with PD. ${ }^{1}$ A hallmark of PD progression is the emergence of walking dysfunction, ${ }^{2}$ which is exacerbated under dual-task conditions. ${ }^{1}$ Because dual tasking during walking requires divided attention and intact executive function through motor-cognitive interplay, cognitive impairments in people with PD contribute to decreased walking performance and deteriorated walking patterns (ie, decreased 
cadence, increased step variability). ${ }^{3}$ Moreover, motor and cognitive impairments should be addressed simultaneously to improve dual tasking in people with PD.

While current medical interventions (ie, medication and deep brain stimulation) address motor symptoms, non-motor symptoms, including cognitive function are inadequately addressed. Non-pharmacological interventions, such as non-invasive brain stimulation techniques are emerging as potential complementary treatment approaches for people with neurodegenerative conditions, such as PD. ${ }^{4}$ Transcranial direct current stimulation (tDCS), a method of non-invasive brain stimulation, is currently being explored as an approach to improve motor and cognitive function. ${ }^{5}$ In healthy individuals, tDCS has demonstrated enhanced motor ${ }^{6}$ and executive functioning ${ }^{7}$ as well as postural control and dual-task walking. ${ }^{8}$ Similarly, motor function (ie, walking, mobility, and balance) and cognitive function improved separately after anodal tDCS in people with PD. ${ }^{9,10}$

However, the tDCS approach appears to drive domainspecific outcomes. Specifically, while anodal tDCS at M1 improved motor but not cognitive function, ${ }^{9}$ electrode placement at the dorsolateral prefrontal cortex (DLPFC) demonstrated improved cognitive function. ${ }^{10}$ Furthermore, tDCS applied to M1 is unlikely to be effective in enhancing dual-task walking. ${ }^{11}$ As such, though tDCS has shown domain-specific improvements in people with $\mathrm{PD}$, the therapeutic potential promoting combined motor and cognitive interplay is undetermined. A recent animal model suggests a bi-hemispheric tDCS approach raises striatum extracellular dopamine levels (cathode over right prefrontal cortex) while supporting functional brain network connectivity (anode over left prefrontal cortex). ${ }^{12}$ We applied this bi-hemispheric approach to people with PD and observed a lessening (though non-significant) of dual-task cost (DTC) during walking after a single tDCS session. ${ }^{13}$ Recent studies suggest anodal tDCS combined with concurrent training (tango dancing, ${ }^{14}$ dynamic balance training ${ }^{15}$ ) may augment beneficial effects on walking and balance compared with tDCS alone. To our knowledge, the concept of tDCS plus a concurrent activity has not yet been explored in dualtask walking in PD. Therefore, our objective in this pilot study was to observe the influence of a single session of bi-hemispheric tDCS paired with a concurrent activity on walking-associated DTC in people with PD. We selected a non-walking aerobic exercise activity (pedaling a stationary bicycle) and a novel activity (playing a video game of golf on $\mathrm{Wii}^{\mathrm{TM}}$ ) as our concurrent tasks to examine whether type of task was consequential to performance.

\section{Materials and methods}

\section{Participants}

Eighteen participants were recruited from a convenience sample to participate in this study from local rehabilitation centers, PD support groups, and by word-of-mouth. Prospective participants were initially screened to determine if they had a diagnosis of PD based on the UK Brain Bank criteria from their movement disorder neurologist, were between ages 40 and 85 years old, and considered medically stable as defined by no pharmaceutical changes within 45 days. The presence of a deep brain stimulator or a pacemaker resulted in exclusion from the study. All participants who met the inclusion criteria provided written informed consent and this study was approved by The Texas Woman's University Institutional Review Board. Disease severity for our participants was determined by the Hoehn and Yahr scale and United PD Rating Scale (UPDRS). Executive function was assessed at baseline for each participant by the Repeatable Battery for the Assessment of Neuropsychological Status (RBANS). ${ }^{16}$ Each participant was tested during medication "ON" times for all conditions. Refer to Figure 1 for details.

\section{Procedure}

Participants received 4 tDCS sessions tDCS $_{\text {Sitting, }} \mathrm{tDCS}_{\text {Bike, }}$ $\mathrm{tDCS}_{\mathrm{Wii}_{\mathrm{i}}}$, $\mathrm{tDCS}_{\text {Sham) }}$ each separated by $7 \pm 2$ days. tDCS sessions were randomized and the sham condition was blinded to participants. For each session, saline-soaked $3 \mathrm{~cm} \times 5 \mathrm{~cm}$ electrode sponges were placed directly on the participants' scalp over the hair with the anodal electrode over the left DLPFC and the cathodal electrode over the right DLPFC. Electrode placement was determined by the 10-20 International System and rechecked at the beginning of each session. For active tDCS sessions ( $\mathrm{tDCS}_{\text {Sitting, }} \mathrm{tDCS}_{\text {Bike, }}$ and $\mathrm{tDCS}_{\mathrm{Wii}}$ ), a constant electrical current of $2 \mathrm{~mA}$ was applied for 20 minutes with a 30 second electrical current ramp at the onset and termination. For the $\mathrm{tDCS}_{\text {Sham }}$ condition, an electrical current of 1 $\mathrm{mA}$ started the first 30 seconds of the stimulation followed by slow tapering to $0 \mathrm{~mA}$ to limit potential sensation bias. During the $\mathrm{tDCS}_{\text {Sitting }}$ and $\mathrm{tDCS} \mathrm{S}_{\text {Sham }}$ sessions, participants were seated without a concurrent activity. During the $\mathrm{tDCS}_{\text {Bike }}$ session, participants pedaled a stationary bicycle at a moderate aerobic intensity concurrent with tDCS stimulation. In order to achieve moderate aerobic intensity, participants were asked to pedal at a work rate consistent with a self-reported intensity level of 12-14 on the Borg Rating of Perceived Exertion Scale. ${ }^{17}$ Physiological responses (heart rate, blood oxygen 


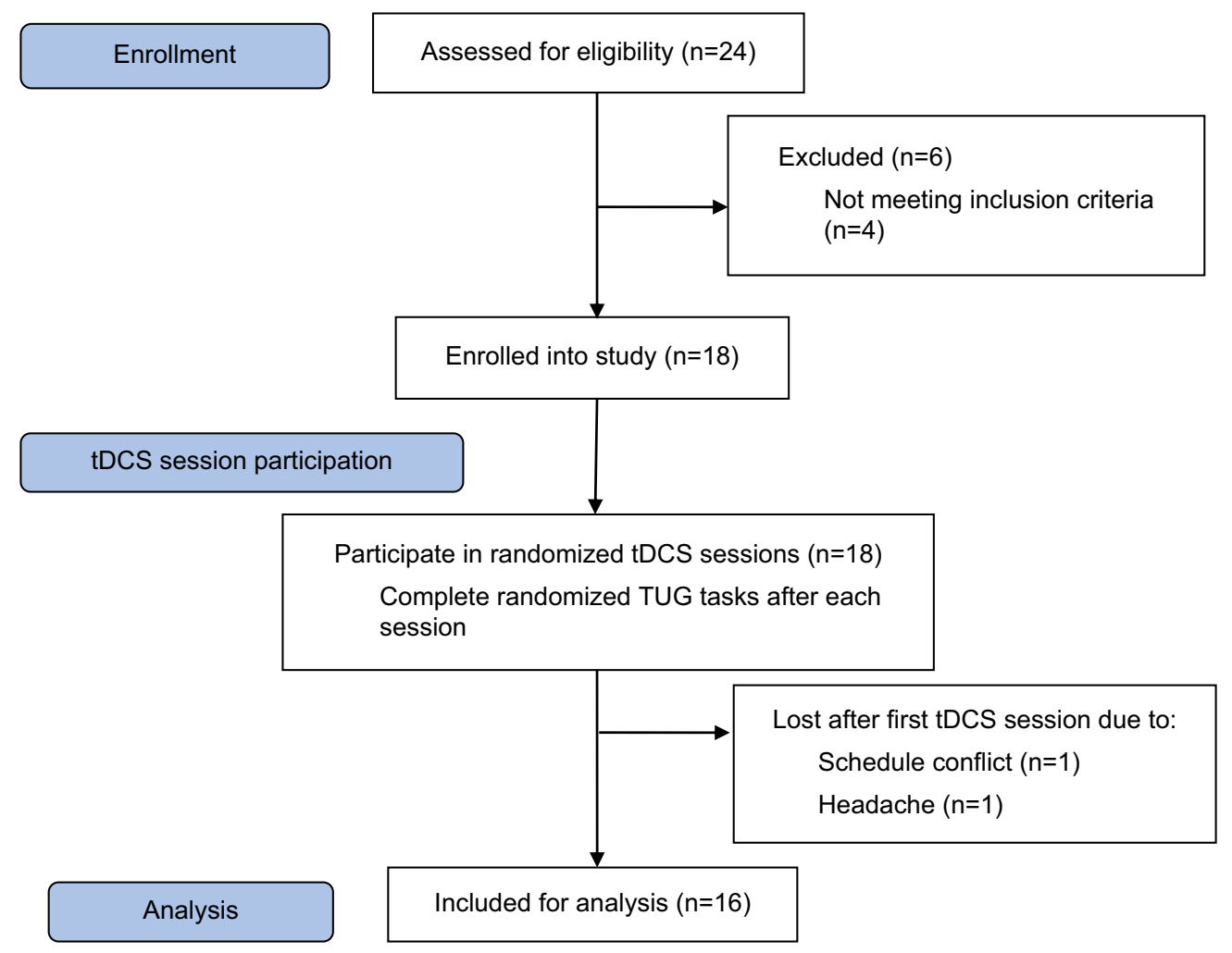

Figure I CONSORT flow diagram.

Abbreviations: tDCS, transcranial direct current stimulation; TUG, Timed Up and Go.

saturation level) were also monitored at baseline and every 5 minutes throughout the session. For the $\mathrm{tDCS}_{\mathrm{Wii}}$ session, participants engaged in a novel activity of a golf video game on the Nintendo Wii game system in a standing or seated position, based on individual tolerance, concurrent with tDCS stimulation. Following each tDCS session, participants performed a single (Timed Up and Go $\left[\mathrm{TUG}_{\text {alone }}\right]$ ) and dual-task gait $\left(\mathrm{TUG}_{\text {motor }}\right.$ and $\mathrm{TUG}_{\text {cognitive }}$ ) activity.

\section{Outcome measures}

We assessed the interplay between motor and cognitive function during walking through single- and dual-task conditions with the use of 3 Timed Up-n-Go (TUG) scenarios: $\mathrm{TUG}_{\text {alone}}$, $\mathrm{TUG}_{\text {motor }}$, and $\mathrm{TUG}_{\text {cognitive. }}{ }^{18}$ In the single attention $\mathrm{TUG}_{\text {alone }}$ task, the participant was instructed to stand up at a normal self-selected pace, walk $3 \mathrm{~m}$, turn $180^{\circ}$, walk back, and sit down. During the $\mathrm{TUG}_{\text {motor }}$, the participant held a full cup of water while performing the TUG task. During the $\mathrm{TUG}_{\text {cognitive }}$, the participant counted backwards by 3 from a randomly chosen number between 50 and 100 while performing the TUG task. Following TUG tasks, participants were instructed to count aloud backwards by 3 in a seated position to serve as a single cognitive alone task for comparison to $\mathrm{TUG}_{\text {cognitive }}$. The time to complete each task was recorded, and the number of correct serial 3 calculations was also recorded during the $\mathrm{TUG}_{\text {cognitive }}$ and cognitive alone tasks. Prior to testing, participants completed 1 practice trial of the $\mathrm{TUG}_{\text {alone }}$ to familiarize the participant with the walking task. All 3 TUG tasks were randomized and performed following each tDCS session under direct supervision of an investigator.

\section{Data analysis}

All statistical analyses were performed using SPSS (Version 24.0). Descriptive statistics were used to analyze participant characteristics, including disease severity and executive function. The impact of adding a secondary task to gait was assessed by performing DTC calculations using the following formula: (single task [s] - dual task [s] / single task [s]) $\times 100$. Because of our small sample size, we used nonparametric statistics for our analysis. Friedman's Two-Way Analysis of Variance by Ranks test was used to compare within subject performance differences of tDCS sessions on single- and 
dual-task TUG conditions and DTC. If Friedman's Two-Way Analysis of Variance by Ranks was significant, pairwise comparisons were utilized to differentiate between conditions.

\section{Results}

Of the 18 participants we recruited, 2 participants withdrew (1 with complaints of a headache after the first session and 1 with a schedule conflict) and were removed from analysis. The remaining 16 participants ( 12 male and 4 female) completed our study with no complaints of discomfort during tDCS protocols. Our participants demonstrated a median Hoehn and Yahr stage of 2 (range $=2-4$ ) and a mean UPDRS total score of 40.31 (motor sub-score=23.44) indicating our participants on average exhibited mild motor impairments. The RBANS mean total scaled score for executive function was 82.69 ranking our participants in the thirteenth percentile with their age-matched peers. Six of our 16 participants reported falling within the previous year $(n=1,>1$ fall per day; $\mathrm{n}=2,>1$ fall per week; $n=3,>1$ fall per month). See Table 1 for participant characteristics.

To determine if a single session of tDCS under different conditions improved dual-task performance during walking in people with $\mathrm{PD}$, we performed a session comparison on single- and dual-task TUG conditions. There were no differences for $\mathrm{TUG}_{\text {alone }}(p=0.738)$ and $\mathrm{TUG}_{\text {motor }}(p=0.807)$ conditions between tDCS sessions. $\mathrm{TUG}_{\text {cognitive }}$ was significant ( $p=0.037$ ) on Friedman's Two-Way Analysis of Variance by

Table I Participant characteristics at baseline $(n=16)$

\begin{tabular}{ll}
\hline Baseline & Mean \pm SD \\
\hline Age (years) & $68.13 \pm 9.76$ \\
Gender (male/female) & $12 / 4$ \\
PD duration (years) & $8.69 \pm 9.76$ \\
UPDRS (total) & $40.31 \pm 18.27$ \\
UPDRS (motor) & $23.44 \pm 9.73$ \\
Schwab and England score (\%) & $87.81 \pm 7.52$ \\
RBANS (total scaled score) & $84.13 \pm 14.83$ \\
\hline
\end{tabular}

Abbreviations: PD, Parkinson's disease; RBANS, Repeatable Battery for the Assessment of Neuropsychological Status; UPDRS, Unified Parkinson's Disease Rating Scale.
Ranks; however, no post hoc pairwise comparisons were significant. As expected, however, regardless of tDCS session, the time necessary to complete dual-task TUG conditions (TUG $_{\text {motor }}$ and TUG $_{\text {cognitive }}$ ) was greater than the single-task TUG condition $\left(\mathrm{TUG}_{\text {alone }}\right)$, indicating an associated cost during dual-task activities. See Table 2 for session comparisons.

To examine the influence of DTC on gait, we calculated the associated cost observed during dual-task activities. As anticipated, DTC was generally larger during the higher complexity $\mathrm{TUG}_{\text {cognitive }}$. Using Friedman's Two-Way Analysis of Variance by Ranks, differences were observed on the DTC for the $\mathrm{TUG}_{\text {cognitive }}$ walking activity $(p=0.010)$, but not on $\mathrm{TUG}_{\text {motor }}(p=0.807)$ or $\mathrm{TUG}_{\text {cognitive }}$ cognitive task $(p=0.754)$. Post hoc pairwise comparisons indicated a difference in DTC in walking between $\mathrm{TUG}_{\text {Bike }}$ and $\mathrm{TUG}_{\mathrm{Wii}}$ conditions $(p=0.007)$ (Figure 2).

\section{Discussion}

People with PD demonstrate greater challenges with walking during associated dual tasks due in part to disease-related deficits and interference in motor-cognitive interplay. ${ }^{2}$ Recently, others have linked the non-pharmacological intervention of anodal tDCS, with or without a concurrent activity, to domain-specific improvements to motor or cognitive function related to anode placement (M1 or DLPFC). ${ }^{9,10,19}$ However, 3 weeks of walking training with M1 anodal tDCS was not beneficial on multi-domain tasks (ie, dual-task walking) for people with PD. ${ }^{11}$ In this current pilot study, we examined if our bi-hemispheric tDCS protocol paired with concurrent activities would reduce DTC with walking in participants with PD. Similar to Scabrun et al, ${ }^{11}$ we observed no significant improvement in performance on the TUG conditions regardless of the tDCS condition or concurrent activity.

However, in order to account for individual baseline walking characteristics, the magnitude of changes in dual-task walking performance may be better expressed through DTC because DTC is related to executive function efficiency. ${ }^{20} \mathrm{In}$ our study, the DTC associated with addition of a secondary task appeared to be influenced by both task complexity as

Table 2 tDCS session outcomes $(n=16)$

\begin{tabular}{|c|c|c|c|c|c|}
\hline \multirow[t]{2}{*}{ Outcome } & tDCS $_{\text {sitting }}$ & tDCS $_{\text {bike }}$ & tDCS $_{w_{\mathrm{ii}}}$ & tDCS $_{\text {sham }}$ & \multirow[t]{2}{*}{$p$-value } \\
\hline & Mean \pm SD & Mean \pm SD & Mean \pm SD & Mean \pm SD & \\
\hline $\mathrm{TUG}_{\text {alone }}(\mathrm{s})$ & $9.49 \pm 2.42$ & $9.38 \pm 3.32$ & $9.15 \pm 2.21$ & $9.44 \pm 3.30$ & 0.738 \\
\hline TUG ${ }_{\text {motor }}(\mathrm{s})$ & $10.88 \pm 3.14$ & | I. $.00 \pm 4.2$ | & $10.6 \mid \pm 2.80$ & | I. $27 \pm 4.4$ | & 0.807 \\
\hline TUG $_{\text {cognitive }}$ (s) & I I. $64 \pm 4.57$ & II. $.20 \pm 5.33$ & $12.11 \pm 3.97$ & I I.38 .4 .44 & $0.037 *$ \\
\hline
\end{tabular}

Note: *Subsequent pairwise comparisons were not significant.

Abbreviations: s, seconds, tDCS, Transcranial Direction Current Stimulation, TUG, Timed Up and Go. 


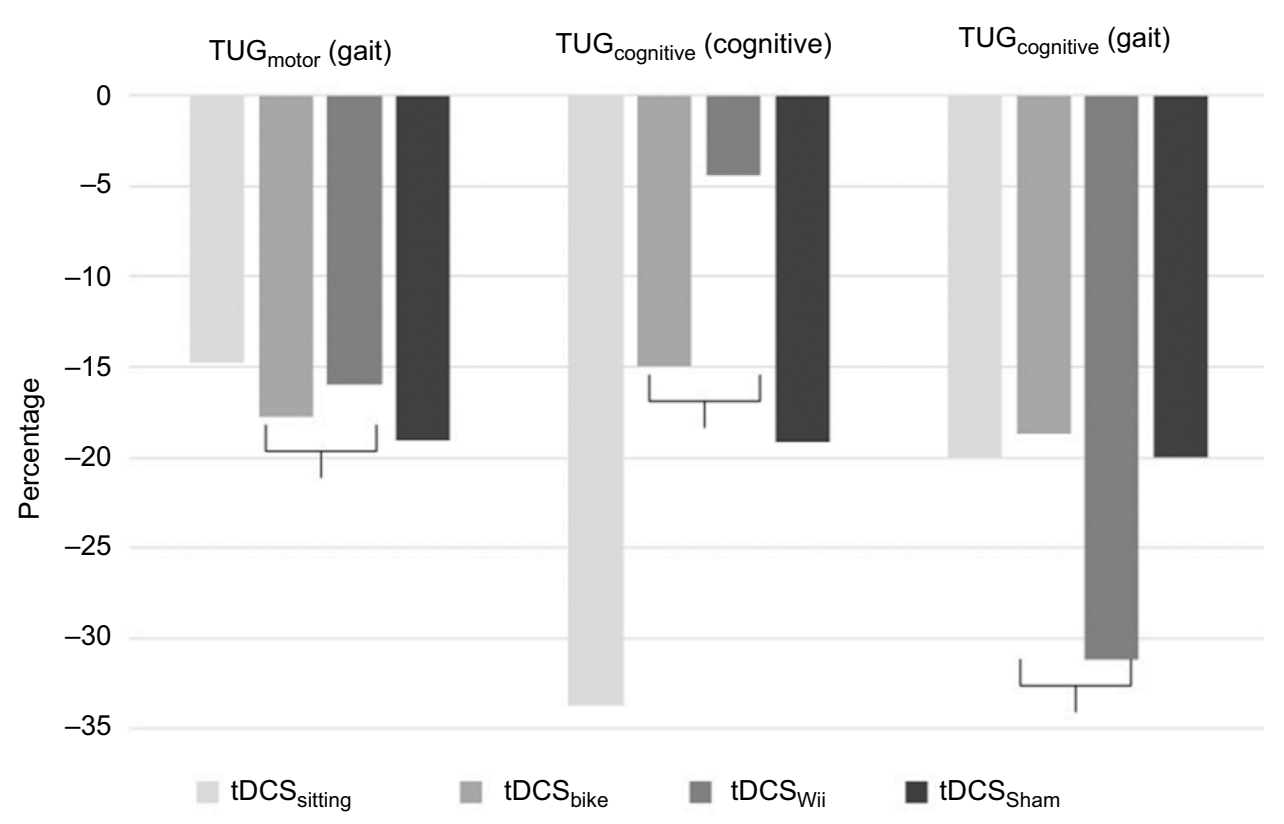

Figure 2 Dual task cost associated with TUG tasks $(n=16)$.

Note: Bracket indicates significant $p$-value $=0.007$.

Abbreviations: tDCS, transcranial direct current stimulation; TUG, Timed Up and Go.

well as tDCS condition (Figure 2). In general, the addition of a more complex secondary task (cognitive distractor) was associated with greater DTC. This finding is consistent with others ${ }^{21}$ and our previous work. ${ }^{13}$ A novel finding is the potential impact of tDCS conditions on DTC. Specifically, tDCS condition may have influenced preferential task prioritization on either the cognitive task or the walking activity. For the $\mathrm{tDCS}_{\mathrm{Wii}}$ condition, our participants walked slower but emphasized performance on the cognitive secondary task during the $\mathrm{TUG}_{\text {cognitive }}$. By contrast, after the $\mathrm{tDCS}_{\text {Sitting }}$ and $\mathrm{tDCS}_{\text {Sham }}$ conditions, our participants were not observed to preferentially select one task over another.

Nearly a decade ago, Bloem et al suggested people with PD emphasize a "posture second" strategy in the presence of a dual task. ${ }^{22}$ By doing so, people with PD prioritize performance on a cognitive task at the expense of balance and gait, which may lead to increased risk of falls. ${ }^{22}$ More recently, Yogev-Seligmann et al proposed an Integrated Model of Task Prioritization, which introduces postural reserve, hazard estimation (cognitive self-awareness), intrinsic individual factors (ie, skilled tasks, mood, personality), and task complexity as key components involved in task prioritization during dual-task walking. ${ }^{23}$ According to this model, the $\mathrm{tDCS}_{\text {Bike }}$ and $\mathrm{tDCS}_{\mathrm{Wii}}$ conditions appear to have altered participants having PD postural reserve or perception of self-awareness. It may be possible that our bi-hemispheric tDCS approach was able to influence both motor (postural reserve) and cognitive processes (hazard estimation) and thus shift task prioritization to focus onto the cognitive task ( $\mathrm{tDCS}_{\mathrm{wii}}$ task) or onto the walking activity $\left(\mathrm{tDCS}_{\text {Bike }}\right)$. We recommend further exploration of the role of tDCS plus concurrent activity to modify task prioritization in an adequately powered trial.

Notwithstanding preferential task prioritization, appreciation of the global cost of dual-task walking (walking speed and cognitive performance) may be better determined by looking at the mutual dual-task effect (Figure 3). As described by Plummer et al, ${ }^{21}$ because decline in performance during dual tasks occurs in both motor and cognitive domains, it is imperative to quantify the mutual dual-task effect to comprehensively understand the sum cost on a dual-task activity. For our participants, visual observation of the mutual dual-task effect reveals the $\mathrm{tDCS}_{\text {Bike }}$ and $\mathrm{tDCS}_{\mathrm{Wii}}$ conditions demonstrated less cumulative cost and appeared potentially advantageous compared with tDCS alone or a sham scenario.

An alternative mitigating explanation for the difference in DTC between tDCS conditions may be fatigue induced from the concurrent tasks. Specifically, fatigue may have limited physical performance or influenced task prioritization during our assessment of motor-cognitive interplay in dual-task walking scenarios. In contrast to the $\mathrm{tDCS}_{\text {Sitting }}$ and $\mathrm{tDCS}_{\text {Sham }}$ conditions, our participants were physically active during the $\mathrm{tDCS}_{\text {Bike }}$ and $\mathrm{tDCS}_{\mathrm{Wii}}$ conditions. Though participants were afforded rest breaks as needed, our participants averaged 20 minutes of stationary bicycle pedaling at moderate intensity $\left(\mathrm{tDCS}_{\text {Bike }}\right)$ or standing to swing a Wii remote control 


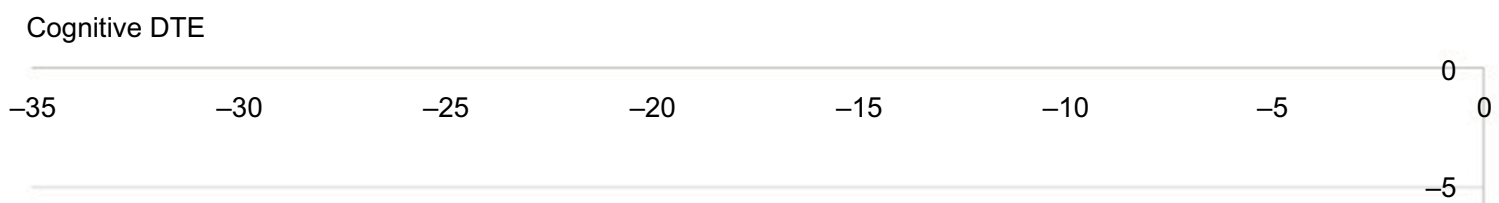

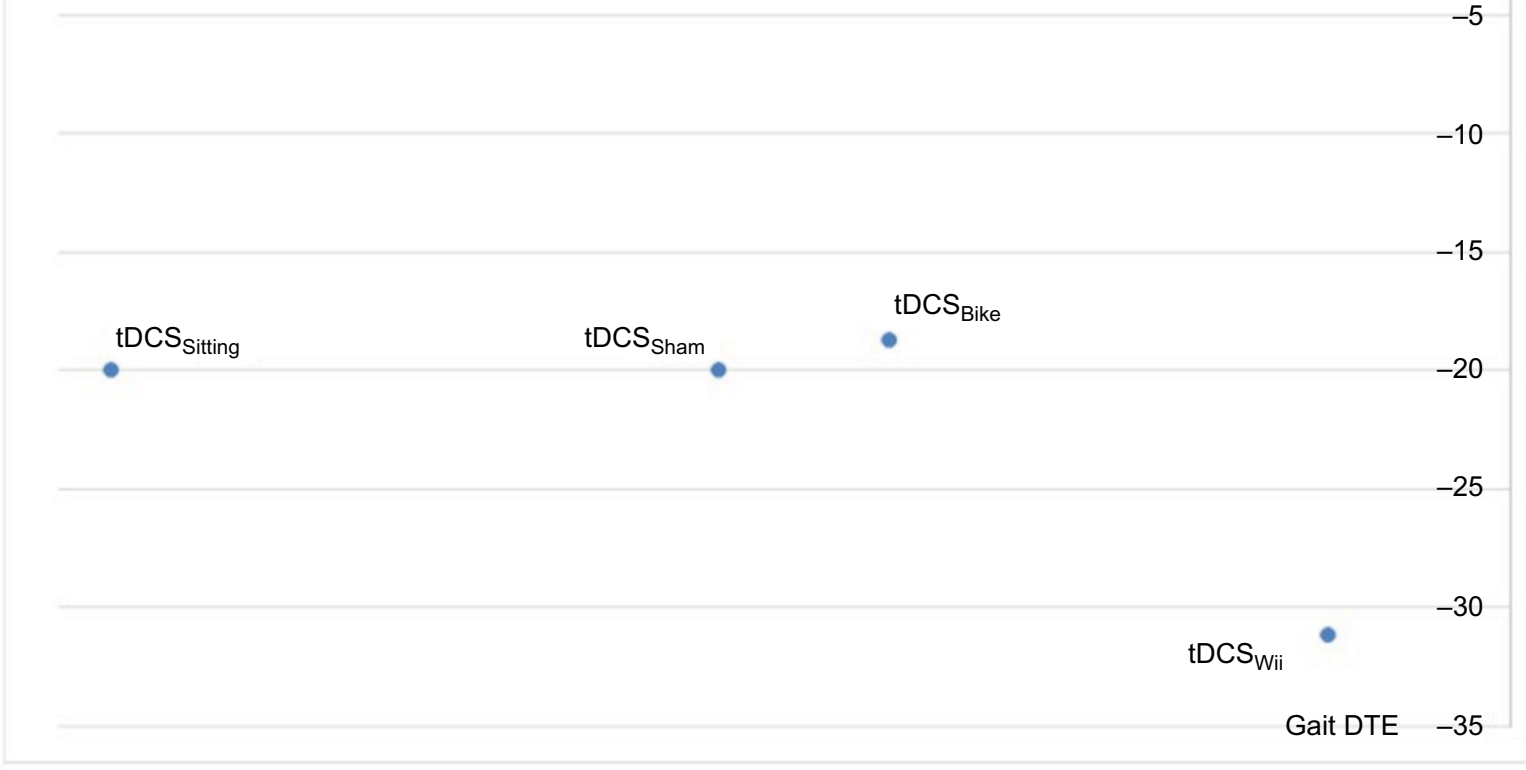

Figure 3 Mutual dual task effects on the TUG ${ }_{\text {cognitive }}$ task $(n=16)$.

Abbreviations: tDCS, transcranial direct current stimulation; DTE, dual-task effect; TUG, Timed Up and Go.

$\left(\mathrm{tDCS}_{\mathrm{wii}}\right)$. It is possible that 20 minutes of physical activity may have fatigued our participants and affected their physical capacity to perform dual-task walking. Unfortunately, we did not intentionally measure fatigue with a specific scale after each condition as this may have added justification to this explanation.

As underscored by Rochester et al, ${ }^{24}$ dual-task walking is complex and the neural correlates and behavioral influences are not completely understood. ${ }^{24}$ Consequently, the mechanism contributing to dual-task walking, and the intervention necessary to maximize performance and minimize associated DTC is unclear. Due to the unique pathology associated with PD, the suitable intervention to improve dual-task gait may not simply require minimizing interferences, but rather adapt the system to correctly prioritize tasks. Our bi-hemispheric tDCS protocol with a concurrent activity may minimize mutual DTC as well as influence task prioritization during walking in people with PD. It should be noted that these changes occurred after a single session and are unlikely to result in lasting behavioral change. Serial tDCS sessions, rather than a single session, may be necessary to drive carryover and lasting behavioral change. Further investigation is needed to determine the optimal number of tDCS sessions needed to achieve improved dual-task walking in people with PD. Moreover, effective task prioritization requires the individual to appropriately prioritize tasks based on the functional scenario. We recommend future studies investigate task prioritization after tDCS under different functional scenarios to determine potential benefits in people with PD.

\section{Limitations}

This study has several limitations. Notably, our small sample size reduces our ability to draw definite conclusions about the use of bi-hemisphere tDCS protocol and the effect on DTC in walking. Furthermore, because our participants demonstrated mild motor deficits on average, our sample is not likely characteristic of all people with PD. Nonetheless, our small sample was not homogeneous in disease duration or disease severity, which likely affected our findings. Each participant performed all testing procedures during their "ON" phase limiting our ability to generalize our results to people with PD during medication "OFF" phases. We utilized the 10-20 International System to determine electrode placement. Although recognized as the standard for scalp electrode positioning, ${ }^{25}$ the $10-20$ International System assumes a consistent correlation between underlying cerebral structures and visible scalp locations and 
does not guarantee correct electrode positioning. It is therefore possible that our electrode placement was not at the DLPFC as intended. Use of neuronavigation tools would potentially improve our electrode placement. Finally, we selected a washout period of $7( \pm 2)$ days because it is considerably longer than the 1 hour reported single tDCS session after-effects. ${ }^{26}$ However, the effects of our active tDCS conditions (tDCS $_{\text {Sitting, }} \mathrm{tDCS}_{\text {Bike, }}$ $\mathrm{tDCS}_{\mathrm{Wii}_{\mathrm{i}}}$ ) may have influenced the $\mathrm{tDCS}_{\text {Sham }}$ condition in some participants.

\section{Conclusion}

Our bilateral brain hemisphere tDCS when paired with concurrent activities did not appear to improve dual-task walking performance in participants with PD. However, examination of DTC suggested our bi-hemisphere tDCS protocol, when paired with a concurrent aerobic or cognitive activity may provide a task-specific benefit (cognitive task bias vs. walking activity bias). Further investigation of bilateral hemisphere tDCS with concurrent activity as a non-pharmacological intervention capable of addressing motor-cognitive interplay during walking in people with PD is necessary.

\section{Acknowledgments}

The authors appreciate the contributions of The Texas Woman's University physical therapy students Caitlyn Dobbs, Bethany Uhl, Naz Gharehkhanian, Dakota McCarty, and Victoria Frels for assisting with data collection. This study was partially funded by the Texas Woman's University Research Enhancement Program.

\section{Author contributions}

$\mathrm{CC}, \mathrm{CS}, \mathrm{JM}$ : conception and design. $\mathrm{CC}, \mathrm{CS}, \mathrm{JM}$ : data acquisition. CC, CS, SA: data analysis. CC, CS, SA, JM: interpretation. $\mathrm{CC}, \mathrm{CS}, \mathrm{SA}, \mathrm{JM}$ : manuscript drafting or critique. $\mathrm{CC}$, CS, SA, JM: manuscript, approval of final version. All authors contributed toward data analysis, drafting and revising the paper, gave final approval of the version to be published, and agree to be accountable for all aspects of the work.

\section{Disclosure}

The authors report no conflicts of interest in this work.

\section{References}

1. Kelly VE, Eusterbrock AJ, Shumway-Cook A. A review of dual-task walking deficits in people with Parkinson's disease: motor and cognitive contributions, mechanisms, and clinical implications. Parkinson's Dis. 2012;2012:918719.

2. Yogev-Seligmann G, Hausdorff JM, Giladi N. The role of executive function and attention in gait. Mov Disord. 2008;23(3):329-342.
3. Weiss A, Herman T, Giladi N, Hausdorff JM. Association between community ambulation walking patterns and cognitive function in patients with Parkinson's disease: further insights into motor-cognitive links. Parkinson's Dis. 2015;2015:547065.

4. Hindle JV, Petrelli A, Clare L, Kalbe E. Nonpharmacological enhancement of cognitive function in Parkinson's disease: a systematic review. Mov Disords. 2013;28(8):1034-1049.

5. Pereira JB, Junqué C, Bartrés-Faz D, et al. Modulation of verbal fluency networks by transcranial direct current stimulation (tDCS) in Parkinson's disease. Brain Stimul. 2013;6(1):16-24.

6. Flöel A. tDCS-enhanced motor and cognitive function in neurological diseases. Neuroimage. 2014;85:934-947.

7. Dockery CA, Hueckel-Weng R, Birbaumer N, Plewnia C. Enhancement of planning ability by transcranial direct current stimulation. JNeurosci. 2009;29(22):7271-7277.

8. Zhou J, Hao Y, Wang Y, et al. Transcranial direct current stimulation reduces the cost of performing a cognitive task on gait and postural control. Eur J Neurosci. 2014;39(8):1343-1348.

9. Broeder et al. Transcranial direct current stimulation in Parkinson's disease: neurophysiological mechanisms and behavioral effects. Neurosci Biobehav Rev. 2015;57:105.

10. Manenti R, Brambilla M, Benussi A, et al. Mild cognitive impairment in Parkinson's disease is improved by transcranial direct current stimulation combined with physical therapy. Mov Disord. 2016;31(5): 715-724.

11. Schabrun SM, Lamont RM, Brauer SG. Transcranial direct current stimulation to enhance dual-task gait training in Parkinson's disease: a pilot RCT. PLoS One. 2016;11(6):e0158497.

12. Leite J, Gonçalves OF, Carvalho S. Facilitative effects of bi-hemispheric tDCS in cognitive deficits of Parkinson disease patients. Med Hypotheses. 2014;82(2):138-140.

13. Swank C, Mehta J, Criminger C. Transcranial direct current stimulation lessens dual task cost in people with Parkinson's disease. Neurosci Lett. 2016;626:1-5.

14. Kaski D, Allum J, Bronstein A, Dominguez R. Applying anodal tDCS during tango dancing in a patient with Parkinson's disease. Neurosci Lett. 2014;568:39-43.

15. Kaski D, Dominguez RO, Allum JH, Islam AF, Bronstein AM. Combining physical training with transcranial direct current stimulation to improve gait in Parkinson's disease: a pilot randomized controlled study. Clinl Rehabil. 2014;28(11):1115-1124.

16. Beatty WW, Ryder KA, Gontkovsky ST, Scott JG, McSwan KL, Bharucha KJ. Analyzing the subcortical dementia syndrome of Parkinson's disease using the RBANS. Arch Clin Neuropsychol. 2003;18(5): 509-520.

17. Borg GA. Psychophysical bases of perceived exertion. Med Sci Sports Exerc. 1982;14(5):377-381.

18. Shumway-Cook A, Brauer S, Woollacott M. Predicting the probability for falls in community-dwelling older adults using the timed up \& go test. Phys Ther. 2000;80(9):896-903.

19. Lattari E, Costa SS, Campos C, de Oliveira AJ, Machado S, Neto GAM. Can transcranial direct current stimulation on the dorsolateral prefrontal cortex improves balance and functional mobility in Parkinson's disease? Neurosci Lett. 2017;636:165-169.

20. Sheridan PL, Solomont J, Kowall N, Hausdorff JM. Influence of executive function on locomotor function: divided attention increases gait variability in Alzheimer's disease. J Am Geriatr Soc. 2003;51(11): 1633-1637.

21. Plummer P, Eskes G, Wallace S, et al; American Congress of Rehabilitation Medicine Stroke Networking Group Cognition Task Force. Cognitive-motor interference during functional mobility after stroke: state of the science and implications for future research. Arch Phys Med Rehabil. 2013;94(12):2565-2574. e6.

22. Bloem BR, Grimbergen YA, van Dijk JG, Munneke M. The "posture second" strategy: a review of wrong priorities in Parkinson's disease. J Neurol Sci. 2006;248(1):196-204. 
23. Yogev-Seligmann G, Hausdorff JM, Giladi N. Do we always prioritize balance when walking? Towards an integrated model of task prioritization. Mov Disord. 2012;27(6):765-770.

24. Rochester L, Galna B, Lord S, Burn D. The nature of dual-task interference during gait in incident Parkinson's disease. Neuroscience. 2014;265: 83-94.
25. Jasper HH. The ten-twenty electrode system of the International Federation. Electroencephalogr Clin Neurophysiol. 1958;10:367-380.

26. Monte-Silva K, Kuo MF, Liebetanz D, Paulus W, Nitsche MA. Shaping the optimal repetition interval for cathodal transcranial direct current stimulation (tDCS). J Neurophysiol. 2010;103(4):1735-1740.

\section{Publish your work in this journal}

Journal of Parkinsonism and Restless Legs Syndrome is an online, open access, peer-reviewed journal. The journal publishes review articles, historical reviews, original research articles, case reports, letters to the editor, clinical teaching cases, neuroradiology highlights, neuropathology highlights, neuropsychiatry highlights, autobiographies, conference

Submit your manuscript here: https://www.dovepress.com/journal-of-parkinsonism-and-restless-legs-syndrome-journal 